\title{
Clinical features and immunohistochemistry profile of biopsy-proven gastric gastrointestinal stromal tumor (GIST): a description of 5 years case series and literature review of GIST
}

\author{
Chuthamas Ongprakobkul', Pakkapon Rattanachaisit', Nachchakorn Kwankajonwong', \\ Duangporn Werawatganon ${ }^{1, *}$, Naruemon Klaikaew², Rungsun Rerknimitr ${ }^{3}$
}

\begin{abstract}
Background: Gastrointestinal stromal tumor (GIST) was the most common mesenchymal tumor of the gastrointestinal tract predominately occurring in the stomach. Although GIST was a rare disease, it was considered to be a lifethreatening malignancy.

Objective: To explore the current status of gastric GIST in Thai patients.

Method: The medical records of patients who were diagnosed with histologically proven gastric GIST from 2012 to 2016 in King Chulalongkorn Memorial Hospital were reviewed.

Results: Of 22 patients, there were 14 (63.6\%) females and 8 (36.4\%) males with the mean age of $62.6 \pm 14.8$ years. The average duration before the first presentation was 12 weeks. The initial symptoms were upper gastrointestinal bleeding $(50.0 \%)$ followed by abdominal pain (31.8\%). Tumor mostly located at the proximal part (fundus and cardia) in $20(90.9 \%)$ patients. At the time of the diagnosis, three patients (13.6\%) had distant metastasis. Of this group, 77.3\% underwent surgical treatment.

Discussion: Although gastric GIST was an uncommon disease, early diagnosis and prompt treatment could save the lives of many patients.
\end{abstract}

Keywords: gastric; gastrointestinal stromal tumor; stomach

\begin{abstract}
A gastrointestinal stromal tumor (GIST) was the most common mesenchymal tumor of the gastrointestinal tract and was originated from Cajal cells or their precursors. This tumor can occur anywhere throughout the gastrointestinal tract from esophagus to rectum; however, it predominately located in the stomach [1]. The main cause of GIST was
\end{abstract}

due to the presence of an abnormal form of tyrosine protein kinase, which is also known as KIT or CD117, resulting in uncontrollable growth of cells in the gastrointestinal tract. The population-based study reported that the annual incidence of GIST was 1.1 cases per 100,000 [2]. Although GIST was a rare disease responsible for $0.1 \%-3 \%$ of all

*Correspondence to: Duangporn Werawatganon, Department of Physiology, Faculty of Medicine, Chulalongkorn University, Bangkok 10330, Thailand, e-mails: duangporn.t@chula.ac.th, dr.duangporn@gmail.com

'Department of Physiology, Faculty of Medicine, Chulalongkorn University, Bangkok 10330, Thailand

2Department of Pathology, Faculty of Medicine, Chulalongkorn University, Bangkok 10330, Thailand

${ }^{3}$ Department of Medicine, Faculty of Medicine, Chulalongkorn University, Bangkok 10330, Thailand

Ә Open Access. @ 2020 Ongprakobkul et al., published by Sciendo. (cc)BY-NC-ND This work is licensed under the Creative Commons Attribution NonCommercial-NoDerivatives 4.0 License. 
gastrointestinal cancer, GIST was considered to be a lifethreatening malignancy [3].

So far, there was not much information about GIST in Thailand; thus the objective of the presented study was to describe the clinical characteristics and immunohistochemistry profile of biopsy-proven gastric gastrointestinal stromal tumor (GIST) from a single tertiary care center.

\section{Material and methods}

This study has been approved for research ethics by the Institutional Review Board of the Faculty of Medicine, Chulalongkorn University (IRB No. 079/61). The study was conducted in compliance with the international guidelines for human research protection as the Declaration of Helsinki. The authors retrospectively reviewed the medical records of patients who visited King Chulalongkorn Memorial Hospital, Bangkok, Thailand. The authors enrolled the patients who were diagnosed with gastric GIST in 5 years from January 2012 to December 2016. The definition of gastric GIST in this study was defined by diagnosis of GIST in ICD-10 diagnosis code system, combined with proven histological demonstration of GIST from the gastric specimen. The medical records were reviewed for patient information such as sex, age, onset of symptoms, chief complaint, alcohol drinking and smoking history, metastasis, and treatment. The location of tumors was explored by endoscopic reports. Histopathological and immunohistochemistry evidences from either endoscopic biopsy or surgical specimen were assessed for confirming the diagnosis. Moreover, Helicobacter pylori (HP) testing was also done in some patients by rapid urease test and/or urea breath test method. Statistical analyses were performed using IBM SPSS Statistics version 23. The data were descriptively analyzed and presented as mean \pm standard deviation (SD).

\section{Results}

Concerning the inclusion criteria of this study, we have recruited 22 patients who were diagnosed with gastric GIST with proven histological evidence. Among this group, there were $14(63.6 \%)$ females and $8(36.4 \%)$ males. The age of this group ranged from 42 to 95 years with the mean age of $62.6 \pm 14.8$ years (Figure 1). The average duration from the onset of symptoms to the hospital was 12 weeks. The initial symptoms which bring patients to the hospital were upper gastrointestinal bleeding $(50.0 \%)$, abdominal

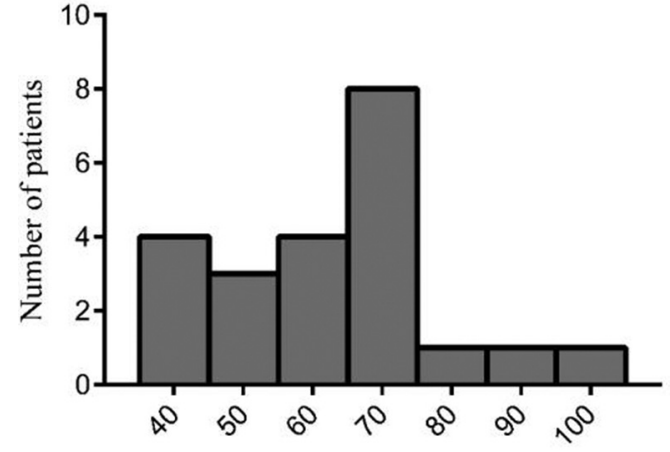

Age at diagnosis (years)

Figure 1. Age distribution of the patients with gastric gastrointestinal stromal tumor.

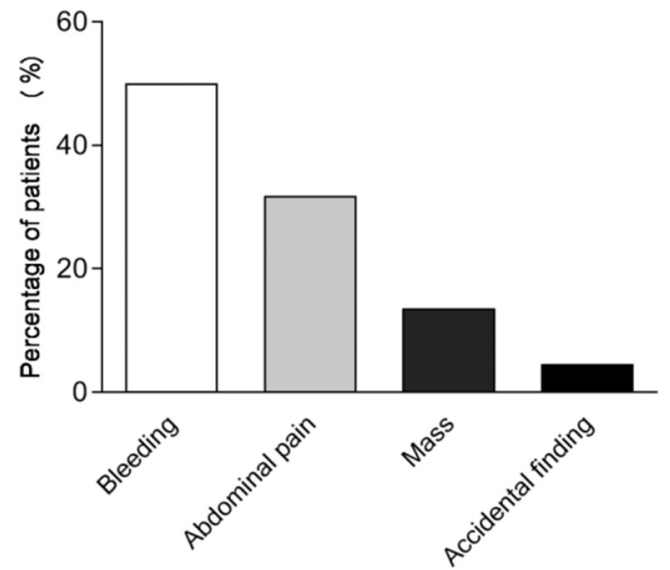

Figure 2. Percentage of patients in each presenting symptom.

pain $(31.8 \%)$, abdominal mass $(13.6 \%)$, and asymptomaticaccidental finding $(4.5 \%)$ (Figure 2). The location of tumor mostly was at proximal part (fundus and cardia) in $20(90.9 \%)$ patients (Table 1). Only one patient had a tumor at antrum and another one was missing data. The immunohistochemistry study of the patients was reported (Table 2). Seven patients of this group received H. pylori testing with rapid urease test and/or urea breath test method and four $(57.1 \%)$ patients had a positive result. Of this group, there were five $(22.7 \%)$ patients and seven $(31.8 \%)$ patients who had a history of smoking and alcohol drinking, respectively (Figure 3). At the time of the diagnosis, three patients $(13.6 \%)$ had distant metastasis. Concerning treatment options, $77.3 \%$ underwent surgical treatment; $9.1 \%$ had got surgical resection combined with chemotherapy; $9.1 \%$ had got only chemotherapy; and $4.5 \%$ had no treatment (Figure 4). The types of surgical treatment are mostly wedge resection or complete excision. 
Table 1. Baseline characteristics, tumor locations, size, mitotic rate of tumors, HP test, treatment, and outcome

\begin{tabular}{|c|c|c|c|c|c|c|c|c|}
\hline Case no & Sex & Age (years) & Tumor location & Size $(\mathrm{cm})$ & Mitotic rate (per 50 HPF) & HP test & Treatment & Outcome \\
\hline 1 & Male & 86 & Cardia & $\mathrm{N} / \mathrm{A}$ & $\mathrm{N} / \mathrm{A}$ & Pos & None & DOD \\
\hline 2 & Male & 68 & Body & 7.0 & 4 & N/A & Surgery & NED \\
\hline 3 & Female & 67 & Cardia & 2.4 & 1 & Pos & Surgery & LDP \\
\hline 4 & Female & 42 & Cardia & 4.5 & 1 & N/A & Surgery & NED \\
\hline 5 & Female & 49 & Cardia & 1.5 & 1 & N/A & Surgery & NED \\
\hline 6 & Male & 46 & $\mathrm{~N} / \mathrm{A}$ & 2.1 & 10 & N/A & Surgery & NED \\
\hline 7 & Female & 72 & Antrum & 2.7 & 2 & Neg & Surgery & NED \\
\hline 8 & Female & 54 & Body & 3.4 & 3 & N/A & Surgery & NED \\
\hline 9 & Female & 59 & Body & 4.0 & $<5$ & N/A & Surgery & NED \\
\hline 10 & Female & 69 & Cardia & 5.0 & 2 & N/A & Surgery & NED \\
\hline 11 & Female & 42 & Fundus & 2.2 & 1 & N/A & Surgery & NED \\
\hline 12 & Male & 71 & Fundus & 4.7 & $<5$ & N/A & Surgery & NED \\
\hline 13 & Female & 59 & Body & 10.8 & 2 & Neg & Surgery & NED \\
\hline 14 & Female & 60 & Body & 4.5 & 3 & N/A & Surgery & NED \\
\hline 15 & Female & 83 & Body & 2.5 & 4 & N/A & Surgery & NED \\
\hline 16 & Male & 42 & Body & 4.0 & $>10$ & N/A & Surgery & NED \\
\hline 17 & Male & 44 & Body & 5.0 & 2 & N/A & Surgery & NED \\
\hline 18 & Female & 95 & Body & 5.5 & 4 & Pos & Surgery & NED \\
\hline 19 & Male & 67 & Fundus & $\mathrm{N} / \mathrm{A}$ & $\mathrm{N} / \mathrm{A}$ & Pos & CMT & LDP \\
\hline 20 & Female & 74 & Body & $\mathrm{N} / \mathrm{A}$ & $\mathrm{N} / \mathrm{A}$ & N/A & CMT & DOD \\
\hline 21 & Male & 68 & Body & 7.5 & 108 & N/A & Surgery + CMT & LDP \\
\hline 22 & Female & 61 & Fundus & 11.0 & 11 & Neg & Surgery + CMT & LDP \\
\hline
\end{tabular}

HP, Helicobacter pylori; Pos, Positive; Neg, Negative; N/A, No available data; CMT, chemotherapy; DOD, died of disease; LDP, local disease progression; NED, no evidence of disease.

\section{Discussion}

In general, GIST equally occurred in both males and females; however, GIST in pediatric patients predominately occurred in girls [4]. In contrast, some studies reported slightly higher incidence rate of GIST in males compared with females [3]. While GIST can affect any age group, several studies demonstrated that this tumor usually develops in older patients, commonly $>50$ years old. Additionally, advanced age was considered to be an etiologic risk factor of GIST $[5,6]$. Similar to this study, the incidence of GIST was reported to be much less in people with age $<40$ years, especially in children and young adults [7]. Moreover, a recent study involving the impact of age and gender on GIST prognosis demonstrated that younger age $(<50$ years) and female gender significantly associated with favorable tumor-related prognosis in terms of diseasespecific survival [8].

Clinical presentations of GIST are associated with the size and location of tumor. Increased size of tumor could aggravate mass-related symptoms, including abdominal discomfort, abdominal pain, and sensation of abdominal satiety. Whereas patients with the small size of tumor are usually asymptomatic [3]. The asymptomatic tumor is frequently identified as an incidental finding by upper endoscopy or other imaging modalities. Symptomatic GIST is usually found either by imaging workup for the presenting symptom or emergent surgery in some cases [9]. Regarding emergency conditions, small bowel was the most common site with bowel ileus, whereas gastrointestinal hemorrhage is the most frequent emergency symptom of gastric GIST [10]. Pathology result from either tissue biopsy or surgical specimen was used for definitive diagnosis [9]. In consistent with several works of literature [11, 12], the authors' findings demonstrated that the most common presenting symptom of gastric GIST was gastrointestinal bleeding followed by abdominal pain. The location of tumor was reported to be associated with various symptoms. Gastrointestinal bleeding and abdominal pain were more common in gastric GIST, whereas jejunal and ileal GIST were frequently presented with acute abdominal symptom [11]. 
Table 2. Immunohistochemistry study of patients

\begin{tabular}{|c|c|c|c|c|c|c|c|c|}
\hline Case no & CD34 & CD117 & DOG1 & S-100 & SMA & MSA & Vimentin & Ki67 \\
\hline 1 & Positive & Positive & & Positive & Positive & & & Positive \\
\hline 2 & Positive & Positive & Positive & Negative & & & Positive & \\
\hline 3 & Positive & Positive & Positive & Negative & Negative & & & \\
\hline 4 & Negative & Negative & Negative & Negative & Positive & Positive & Positive & \\
\hline 5 & Positive & Positive & & Negative & Negative & & Positive & Negative \\
\hline 6 & Positive & Positive & & Negative & Negative & & & \\
\hline 7 & & Positive & & & & & & Negative \\
\hline 8 & & Positive & Positive & Negative & Negative & & & \\
\hline 9 & Positive & Positive & Positive & Negative & Negative & & & Positive \\
\hline 10 & & Positive & Positive & & & & & Positive \\
\hline 11 & & & Positive & & & & & \\
\hline 12 & Positive & Negative & Negative & Negative & Negative & Negative & Positive & \\
\hline 13 & Positive & Positive & Positive & Negative & Negative & & & Negative \\
\hline 14 & & Positive & Positive & & & & Positive & Negative \\
\hline 15 & Positive & Positive & Positive & & & & & \\
\hline 16 & & Positive & Positive & & & & & Positive \\
\hline 17 & Positive & Positive & Positive & Negative & Negative & Negative & & Positive \\
\hline 18 & Positive & Positive & Positive & Negative & Negative & & & \\
\hline 19 & & Positive & & & & & & \\
\hline 20 & Positive & Positive & Positive & Negative & Negative & Negative & & Positive \\
\hline 21 & Positive & Positive & Positive & Negative & Negative & Negative & Positive & Positive \\
\hline 22 & Positive & Positive & & Negative & & & & Positive \\
\hline
\end{tabular}

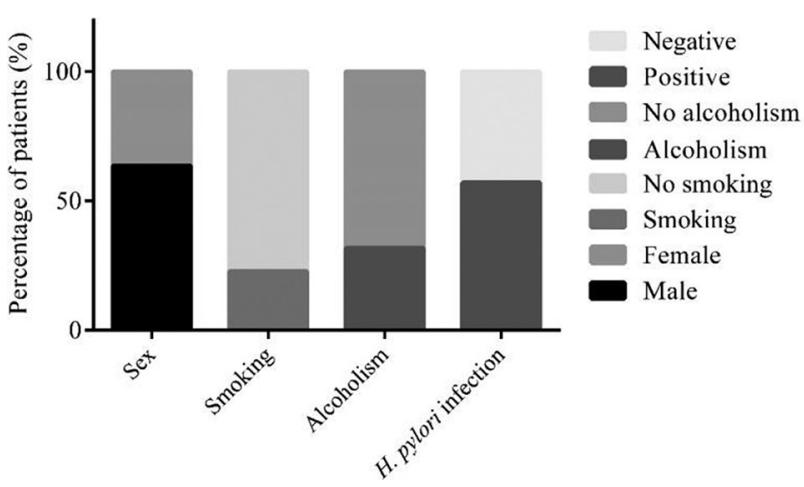

Figure 3. Patient characteristics.

In contrast to gastric cancer, environmental exposures including diet, HP, and smoking appeared to have no strong association with GIST. HP could be a possible carcinogenic candidate related to the pathogenesis of GIST [13]. A pathological review of stomach biopsy from patients with and without GIST found that in patients with GIST, $62.9 \%$ were positive for HP, and only $30.0 \%$ of patients who did not have GIST were positive for HP $(P=0.013)$ [14]. In our

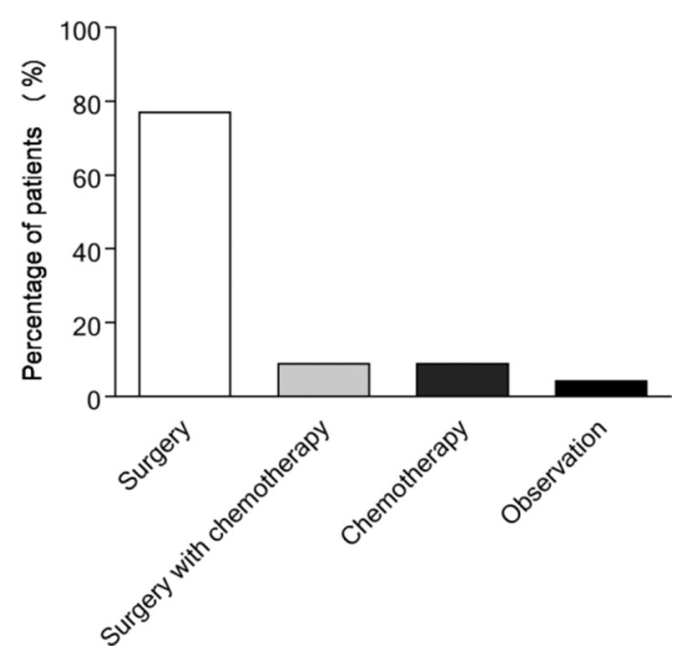

Figure 4. Percentage of patients in each treatment option.

series, four $(57.1 \%)$ out of seven patients who were tested for HP had HP infection.

In addition to advanced age $[5,6]$, the associated risk factor of GIST was genetic syndrome. Although most GISTs 
were sporadic, in rare cases, GIST resulted from an inherited gene mutation in family members. Activation of mutation on KIT or PDGFRA gene was believed to be mostly accounted for a pathogenesis of familial and sporadic GIST [15]. The hallmark diagnostic marker of GIST is positive CD117 immunohistochemical staining, which is a representative of receptor tyrosine kinase KIT overexpression. The KIT expression is positive in $>90 \%$ of GISTs; however, $4-5 \%$ of cases are KIT-negative [16, 17]. The mutation analysis of KIT or PDGFRA is needed to confirm the diagnosis of GIST [18]. Furthermore, GIST was also found to be associated with other genetic diseases that do not have KIT or PGDFRA mutations including neurofibromatosis type 1 (NF1) and Carney-Stratakis syndrome (CSS). NF1, also known as von Recklinghausen disease, is an inherited autosomal dominant disease that is characterized by multiple café au lait spots, Lisch nodules, freckling, and neurofibromas [19]. GIST was reported to develop 150 times more frequently in patients with NF1 than in the general population [20]. It was hypothesized that NF1 resulted from a loss-of-function of tumor suppressor gene leading to the inactivation of neurofibromin that regulated cell proliferation and cell differentiation. Whereas CSS or hereditary GIST paraganglioma syndrome was caused by a loss of succinate dehydrogenase subunit B (SDHB) expression [19]. Other immunohistochemical markers that are positive in GIST regardless of KIT/PDGFRA mutational status are DOG-1 and PKC-theta (protein kinase C theta) [21].

Regarding prognosis of tumor, gastric GIST generally had more favorable prognosis than intestinal GIST. Gastric GIST with size $\leq 10 \mathrm{~cm}$ and $\leq 5$ mitoses per 50 high-power field (HPF) had a low risk for metastasis, whereas those with size $>5 \mathrm{~cm}$ and $>5$ mitoses per $50 \mathrm{HPF}$ had a high risk for metastasis [1]. In our series, there were two patients with tumor size $>5 \mathrm{~cm}$ and $>5$ mitoses per $50 \mathrm{HPF}$. These patients had disease progression after surgery requiring imatinib treatment. Contrary to intestinal GIST, tumor with size $>5 \mathrm{~cm}$ independent of mitotic rate had at least moderate risk for metastasis, and all tumors $>5$ mitoses per 50 HPF had a high metastatic risk. Intestinal GIST with size $\leq 5 \mathrm{~cm}$ and $\leq 5$ mitoses per $50 \mathrm{HPF}$ had a low metastatic risk [1]. With respect to this presented data of gastric GIST, the majority of cases had no distant metastasis at the time of diagnosis. Additionally, a recent study in Asia [22] demonstrated that non-gastric disease location, larger tumor size, higher mitotic rate, and aggressive behavior including tumor metastasis or local invasion before treatment are associated with poor relapse-free survival. Whereas larger tumor size, higher mitotic rate, aggressive behavior, negative smooth muscle actin expression, and palliative resection significantly played a vital role in poor overall survival.
The current guidelines for the treatment of GIST referred to complete surgical resection as the first step of treatment for primary GIST $[23,24]$. The presented data were consistent with the previous study which stated that surgery was the standard choice of treatment for primary GIST and the two most common types of surgeries were wedge resection and complete resection. Laparoscopic wedge resection was suggested for gastric GIST having size $<5 \mathrm{~cm}$, whereas complete resection was recommended with endoscopic enucleation in the presence of pseudocapsule [25]. Lymph node resection was not indicated unless lymph node metastasis was clinically evident $[4,26]$. TKI imatinib, which was the first line of chemotherapy, had a role in the treatment of high-risk GIST. The authors' study found that chemotherapy was given in $18.2 \%$ of patients. Chemotherapy may be given as neoadjuvant therapy to turn inoperable GIST to resectable GIST. It can also be given as adjuvant therapy to control disease in palliation for metastatic GIST after resection and prevent recurrence in high-risk GIST [4, 23]. Imatinib-resistant GISTs may be considered to give sunitinib as the second-line chemotherapy and regorafenib as the third-line chemotherapy. All 22 GIST patients did not have radiotherapy. This finding was consistent with previous literature stating that radiotherapy had little role in the management of GIST. GIST was considered to be relatively radio-resistant, and radiotherapy can damage vital organs close to GIST [27].

There were some limitations to this study. First, there was some missing information because of the method used to collect data, which was a retrospective review from medical records. Second, as GIST was an uncommon disease in Thailand, the interpretation of results may be limited because of the small sample size.

\section{Conclusion}

Although GIST was an uncommon disease, it can occur in any gender and age groups. Moreover, the clinical manifestation of GIST was varying from asymptomatic to an emergency condition. GIST was mostly found in the stomach and the majority of gastric GIST presented with gastrointestinal bleeding. Because of the smaller tumor size, gastric GIST tended to have favorable prognosis than intestinal GIST. Thus, early diagnosis and proper treatment would save lives and improve prognostic outcome of many patients, especially who presented with small tumor and GIST-related emergencies. Surgical resection is still a primary treatment of GIST; however, chemotherapy also played a vital role in the management of GIST, especially in high-risk cases. 
The authors found some literature that stated about clinical and epidemiological knowledge of GIST. After reviewing them, we found that most of the studies reported the incidence of disease, patient characteristics, prognosis, and treatment. GIST is mesenchymal tumors that arise at the gastrointestinal tract. The predominant age of patients having GIST was $>40$ years. Although some of the GISTs were related to gene mutation, such as KIT or PDGFRA gene, most were sporadic. GIST usually presents with nonspecific symptoms (i.e., bloating and early satiety), gastrointestinal bleeding, abdominal pain, and obstruction. Even though there were some literature reporting about GIST, research conducted in Thailand was still limited.

This presented study provided clinical information about GIST in Thailand. When compared to the studies conducted in some western and eastern countries, the clinical features were consistent with theirs. This study found that the predominant age of patients was $>40$ years. The presenting symptoms were gastrointestinal bleeding and abdominal pain. The treatment of choice was surgery, which was consistent with the western guideline. Although there were some literature about gastric GIST in western or other eastern countries, the data of Thai patients were still limited. We suggested further study of gene mutation and prognostic analysis in the Thai population to advance understanding of GIST in Thai patients.

Author contributions. DW and RR contributed substantially to the conception and design of this study. CO, PR, NKw, DW, and NK contributed substantially to the acquisition of data. $\mathrm{CO}, \mathrm{PR}, \mathrm{DW}$, and $\mathrm{NK}$ analyzed and interpreted the data. $\mathrm{CO}$, NKw, and DW drafted the manuscript. PR, NK, and RR contributed substantially to its critical revision. All the authors approved the final version submitted for publication and take responsibility for the statements made in the published article.

Conflict of interest statement. The authors have each completed and submitted an International Committee of Medical Journal Editors Uniform Disclosure Form for Potential Conflicts of Interest. Neither of the authors discloses any potential or actual conflict of interest. No financial or nonfinancial benefits have been or will be received from any party related directly or indirectly to the subject of this article.

Data sharing statement. The datasets generated or analyzed during the current study are available from the corresponding author on reasonable request.

\section{References}

[1] Miettinen M, Lasota J. Gastrointestinal stromal tumors: pathology and prognosis at different sites. Semin Diagn Pathol. 2006; 23:70-83.
[2] Ducimetiere F, Lurkin A, Ranchere-Vince D, Decouvelaere AV, Peoc'h M, Istier L, et al. Incidence of sarcoma histotypes and molecular subtypes in a prospective epidemiological study with central pathology review and molecular testing. PloS One. 2011; 6:e20294.

[3] El-Menyar A, Mekkodathil A, Al-Thani H. Diagnosis and management of gastrointestinal stromal tumors: an up-to-date literature review. J Can Res Ther. 2017; 13:889-900.

[4] Bamboat ZM, Dematteo RP. Updates on the management of gastrointestinal stromal tumors. Surg Oncol Clin N Am. 2012; 21:301-16

[5] Yan BM, Kaplan GG, Urbanski S, Nash CL, Beck PL. Epidemiology of gastrointestinal stromal tumors in a defined Canadian Health Region: a population-based study. Int J Surg Pathol. 2008; 16:241-50.

[6] Wang ZH, Liang XB, Wang Y, Ma GL, Qu YQ, Tian XW. [Epidemiology survey of gastrointestinal stromal tumor in Shanxi Province in 2011]. Zhonghua yi xue za zhi. 2013; 93:2541-4.

[7] Miettinen M, Majidi M, Lasota J. Pathology and diagnostic criteria of gastrointestinal stromal tumors (GISTs): a review. Eur J Can. 2002; 38(Suppl 5):S39-51.

[8] Kramer K, Knippschild U, Mayer B, Bogelspacher K, Spatz H, Henne-Bruns D, et al. Impact of age and gender on tumor related prognosis in gastrointestinal stromal tumors (GIST). BMC Cancer. 2015; 15:57.

[9] Oppelt PJ, Hirbe AC, Van Tine BA. Gastrointestinal stromal tumors (GISTs): point mutations matter in management, a review. J Gastro Oncol. 2017; 8:466.

[10] Ucar AD, Oymaci E, Carti EB, Yakan S, Vardar E, Erkan N, et al. Characteristics of Emergency Gastrointestinal Stromal Tumor (GIST). Hepatogastroenterology. 2015; 62:635-40.

[11] Caterino S, Lorenzon L, Petrucciani N, Iannicelli E, Pilozzi E, Romiti A, et al. Gastrointestinal stromal tumors: correlation between symptoms at presentation, tumor location and prognostic factors in 47 consecutive patients. World J Surg Oncol. 2011; 9:13.

[12] Sorour MA, Kassem MI, Ghazal Ael H, El-Riwini MT, Abu Nasr A. Gastrointestinal stromal tumors (GIST) related emergencies. Int J Surg. 2014; 12:269-80.

[13] Luo J-M, Cao F-L, Meng C, Lin L-J, Ma S-Q, Peng S-H, et al. Clinicopathological and molecular characteristics of synchronous gastric adenocarcinoma and gastrointestinal stromal tumors. Sci Rep. 2017; 7:12890.

[14] Raza MA, Shahab AS, Mazzara P. Gastrointestinal stromal tumors: association with Helicobacter pylori gastritis. Am J Clin Pathol. 2012; 138:A351.

[15] Gopie P, Mei L, Faber AC, Grossman SR, Smith SC, Boikos SA. Classification of gastrointestinal stromal tumor syndromes. Endocr Relat Cancer. 2018; 25:R49-58.

[16] Emile JF, Théou N, Tabone S, Cortez A, Terrier P, Chaumette MT, et al. Clinicopathologic, phenotypic, and genotypic characteristics of gastrointestinal mesenchymal tumors. Clin Gastroenterol Hepatol. 2004; 2:597-605.

[17] Miettinen M, Lasota J. Gastrointestinal stromal tumors - definition, clinical, histological, immunohistochemical, and molecular genetic features and differential diagnosis. Virchows Archiv. 2001; 438:1-12.

[18] Heinrich MC, Corless CL, Duensing A, McGreevey L, Chen CJ, Joseph N, et al. PDGFRA activating mutations in gastrointestinal stromal tumors. Science. 2003; 299:708-10.

[19] Wang JH, Lasota J, Miettinen M. Succinate dehydrogenase subunit $\mathrm{B}$ (SDHB) is expressed in neurofibromatosis 1-associated gastro- 
intestinal stromal tumors (GISTs): implications for the SDHB expression based classification of GISTs. J Can. 2011; 2:90.

[20] Bajor J. Gastrointestinal stromal tumours in patients with type 1 neurofibromatosis. Clin Exp Med J. 2009; 3:247-54.

[21] Novelli M, Rossi S, Rodriguez-Justo M, Taniere P, Seddon B, Toffolatti L, et al. DOG1 and CD117 are the antibodies of choice in the diagnosis of gastrointestinal stromal tumours. Histopathology. 2010; 57:259-70.

[22] Yang ML, Wang JC, Zou WB, Yao DK. Clinicopathological characteristics and prognostic factors of gastrointestinal stromal tumors in Chinese patients. Oncol Lett. 2018; 16:4905-14.
[23] Lim KT, Tan KY. Current research and treatment for gastrointestinal stromal tumors. World J Gastroenterol. 2017; 23:4856.

[24] Maki RG. Gastrointestinal stromal tumors (GIST) and their management. Gastrointest Cancer Res. 2007; 1(4 Suppl 2):S81.

[25] Lanke G, Lee JH. How best to manage gastrointestinal stromal tumor. World J Clin Oncol. 2017; 8:135.

[26] Akahoshi K, Oya M, Koga T, Shiratsuchi Y. Current clinical management of gastrointestinal stromal tumor. World J Gastroenterol. 2018; 24:2806.

[27] Din OS, Woll PJ. Treatment of gastrointestinal stromal tumor: focus on imatinib mesylate. Ther Clin Risk Manag. 2008; 4:149. 